\title{
Enseñar y aprender ciencias. Nuevos escenarios para la interacción entre docentes y estudiantes ${ }^{1}$
}

María Gabriela Lorenzo

Universidad de Buenos Aires, Argentina

Centro de Investigación y Apoyo a la Educación Científica (Ciaec), Argentina

Consejo Nacional de Investigaciones Científicas y Técnicas (Conicet), Argentina

glorenzo@ffyb.uba.ar

orcid.org/oooo-0002-9957-8392

\section{Resumen}

La universidad actual ha sido atravesada por profundos cambios sociales y culturales que plantean nuevas configuraciones y problemáticas que necesitan ser incorporadas como objetos de análisis a las agendas educativas. De este modo, las prácticas pedagógicas de nivel universitario se han convertido en un campo emergente para la investigación didáctica, dado que es aqui donde se concretan las interacciones entre docentes y estudiantes con el fin de construir conocimiento de manera conjunta. El objetivo de este trabajo es una invitación para reflexionar sobre ciertos rasgos de la educación universitaria, particularmente en carreras profesionales vinculadas a las ciencias naturales y experimentales, que interpelan tanto los aprendizajes como la formación pedagógica de sus profesores. Para ello, la discusión se inicia con una descripción de estos nuevos escenarios para enseñar y aprender ciencias en la universidad, para luego debatir sobre la necesidad de una formación pedagógica formal para este colectivo de docentes. Finalmente, esbozamos algunos marcos teóricos vigentes (competencias docentes, conocimiento didáctico del contenido, lenguaje cientifico) que consideramos relevantes a la hora de atender a estas nuevas problemáticas. Concluimos con el planteo de algunos interrogantes que señalan posibles rumbos para este incipiente y fértil campo de investigación, la didáctica de las ciencias de nivel superior.

\section{Palabras clave}

Enseñanza superior; enseñanza de las ciencias; Competencias del docente; Formación de docentes en activo; Práctica pedagógica (Fuente: Tesauro de la Unesco).

$1 \quad$ Este trabajo fue realizado en el marco de los proyectos Ubacyt 20020130100073BA (2014-2017) y Conicet-PIP 11220130100609 CO (2014-2016)

Recepción: 2016-08-02 | Envío a pares: 2016-10-25 | Aceptación por pares: 2017-01-27| Aprobación: 2017-02-01 DOI: 10.5294/edu.2017.20.2.5

Para citar este artículo / To reference this article / Para citar este artigo

Lorenzo, M. G. (2017). Enseñar y aprender ciencias. Nuevos escenarios para la interacción entre docentes y estudiantes. Educación y Educadores, 20(2), 249-263. DOI: 10.5294/edu.2017.20.2.5 


\title{
Science Teaching and Learning: New Scenarios for Interaction between Teachers and Students
}

\begin{abstract}
Today's universities are wrought with profound social and cultural changes that pose new alignments and problems that need to be included as subjects of analysis in educational agendas. University-level teaching practices have become an emerging field for didactic research, since it is there that interaction between teachers and students materializes in order to build knowledge together. In terms of its objective, this work is an invitation to reflect on certain aspects of university education, particularly in professional careers associated with the natural and experimental sciences, aspects that address both learning and pedagogical training for science teachers. To that end, the discussion begins with a description of these new scenarios for teaching and learning science in universities, and goes on to discuss the need for formal pedagogical training for this group of teachers. The article outlines several existing theoretical frameworks (teaching competences, didactic knowledge of content, scientific language) that are considered relevant when dealing with these new problems. It concludes by posing several questions that indicate possible paths for this incipient and fertile field of research; namely, the teaching of science in higher education.
\end{abstract}

Key words

Higher education; science education; teacher's skills; active teacher training; practice teaching (Source: Unesco Thesaurus). 


\section{Ensinar e aprender ciências. Novos cenários para a interação entre docentes e estudantes}

\section{Resumo}

A universidade atual passou por profundas mudanças sociais e culturais que expõem novas configurações e problemáticas que precisam ser incorporadas como objetos de análise às agendas educativas. Assim, as práticas de nivel universitário converteram-se em um campo emergente para a pesquisa didática, já que é aqui onde se concretizam as interações entre docentes e estudantes, com o propósito de construir conhecimento de maneira conjunta. O objetivo deste trabalho é um convite para a reflexão sobre certos traços da educação universitária, particularmente em carreiras profissionais vinculadas às ciências naturais e experimentais, que interpelam tanto as aprendizagens como a formação pedagógica dos seus professores. Para isso, a discussão se inicia com uma descrição desses novos cenários para ensinar e aprender ciências na universidade e então debater sobre a necessidade de uma formação pedagógica formal para esse coletivo de docentes. Por fim, esboçamos alguns marcos teóricos vigentes (competências docentes, conhecimento didático do conteúdo, linguagem científica) que consideramos relevantes no momento de tratar dessas novas problemáticas. Concluímos com a abordagem de alguns questionamentos que assinalam possiveis rumos para esse incipiente e fértil campo de pesquisa, a didática das ciências de nivel superior.

\section{Palavras-chave}

Ensino superior; ensino das ciências; competências do docente; formação de docentes em atividade; Prática pedagógica (Fonte: Tesauro da Unesco). 


\section{La universidad como escenario de enseñanza y aprendizaje}

A la hora de decidir una carrera universitaria o de planificar una profesión para nuestro futuro, el nuevo milenio ofrece un sinfín de alternativas y la libertad de elegir o de cambiar las elecciones sin que nada grave ocurra. Las posibilidades de comunicación, otrora impensadas, la cantidad y variedad de información disponible a un clic de distancia y la incertidumbre sobre el devenir de las cosas, atraviesan la cotidianidad de nuestras sociedades, incluida la formación universitaria.

A pesar de su origen medieval y su resistencia a los cambios vertiginosos de la sociedad en la que está inmersa, la universidad ha sabido perdurar en el tiempo plasmando transformaciones y adaptaciones a su propio paso. Debemos reconocer que, desde entonces, se ha sostenido como un pilar por todos respetado y es la institución a la que se le confía la formación de los profesionales que, desde diferentes ámbitos de desempeño, contribuyen al desarrollo de una nación. Sin embargo, los acelerados cambios sociales, culturales y tecnológicos de las últimas décadas han modificado el paradigma de la formación universitaria (Monereo y Pozo, 2003) al punto tal que los jóvenes de hoy no se detienen ante raigambres preestablecidas, sino que a la hora de elegir una carrera muchos de ellos buscan opciones y propuestas flexibles, alternativas que, a diferencia de las carreras clásicas, se ajusten a su particular manera de concebir el mundo.

Particularmente en Argentina, la reciente condición de obligatoriedad de la escuela secundaria ha llevado a una revisión profunda de sus metas y objetivos. Así, la educación se ha planteado la promoción del desarrollo integral y crítico del ciudadano, con una formación en ciencias ${ }^{2}$ orientada

2 Por razones prácticas, en este texto emplearemos el término ciencia como genérico para referirnos a las ciencias naturales y experimentales vinculadas a la química, la física y la biología como marco de referencia. Esto no acota de ninguna manera la concepción de ciencia para otras disciplinas naturales y sociales. principalmente hacia una apropiada alfabetización científica en lugar de priorizar la histórica misión propedéutica de preparación para continuar los estudios en la universidad.

Estas innovaciones en la educación media han provocado, consecuentemente, que lleguen a la universidad nuevas generaciones de estudiantes de perfiles muy diversos y con formaciones heterogéneas, que no siempre se amoldan a las expectativas de las universidades más tradicionales. En general, la adaptación a la vida universitaria no es fácil y muchos estudiantes demoran más tiempo del esperado para finalizar su carrera o, en el peor de los casos, abandonan sus estudios. Estas situaciones, que suelen ser rotuladas como fracasos, ocasionan la preocupación de las generaciones de adultos, ya sean padres, profesores o autoridades académicas. Sin embargo, arraigadas como están las prácticas de enseñanza provenientes de una concepción heredada de la ciencia, a la universidad se le hace difícil encontrar estrategias para lograr una enseñanza masiva, inclusiva y de calidad.

Esta nueva cultura o forma de entender la vida centrada en lo inmediato, atravesada por la incertidumbre y propensa a los cambios, podría ser una de las razones que impacta sobre todo en las carreras universitarias más consagradas y, entre ellas, a las vinculadas al campo de las ciencias naturales. Sin reconocer fronteras, una tendencia generalizada es la disminución de la cantidad de jóvenes interesados en realizar estudios de carreras como ingeniería, bioquímica o licenciatura en física — quizá porque son consideradas como carreras largas y difíciles (y suelen serlo)-, como lo demuestran las estadísticas sobre el abandono y las demoras en el tránsito curricular.

Hasta aquí hemos considerado las transformaciones que han sufrido los perfiles del estudiantado universitario como un primer punto para tener en cuenta al pensar sobre los nuevos escenarios en donde se realiza la interacción entre los estudiantes 
y los docentes. Un segundo aspecto al que aludiremos a continuación para completar nuestra idea es el de los contenidos.

La calidad de la formación universitaria se basa en la especificidad de los contenidos que abarca, correspondientes a una determinada carrera o profesión. Desde este punto de vista, muchos de los contenidos incluidos en los currículos universitarios poseen elevados niveles de abstracción y de complejidad, los cuales representan una nueva forma de entender el aprendizaje de las ciencias. Esta particular selección de contenidos, difícilmente haya sido popularizada por medios alternativos como textos o documentales televisivos del Discovery Channel o divulgados por Youtube y, por tanto, no se encontrarían disponibles como conocimientos previos de los alumnos, ni siquiera como concepciones alternativas, y, no obstante, deben ser desarrollados apropiadamente para promover las competencias profesionales en los futuros egresados. Si, además, a estas circunstancias le sumamos la necesaria actualización de las asignaturas universitarias debidas al crecimiento incalculable de los conocimientos disciplinares, entenderemos por qué los currículos son de tipo sedimentario y muestran, siguiendo a Monereo y Pozo (2003), una inflación de contenidos que razonablemente no pueden ser enseñados en el tiempo pretendido.

Entonces, si combinamos estas nuevas generaciones de estudiantes con esta cultura universitaria poco flexible y cargada de contenidos, podremos empezar a vislumbrar las graves problemáticas a las que se enfrenta hoy la educación universitaria.

Si, para profundizar nuestro análisis, lo acotamos al caso particular de las disciplinas que nos convocan, además de la cantidad y calidad de los contenidos, debemos incorporar los distintos escenarios en los que se despliega la enseñanza. Más allá de las clases convencionales de sillas y pizarra en donde se desarrollan teorías y ejercicios, se destacan las clases prácticas en los laboratorios, consideradas como un ámbito complejo (Hofstein y Mamlok-Naaman, 2007; Reid y Shah, 2007; Seré, 2002). En este contexto, sería esperable un rol protagónico del estudiante con explicaciones del profesor diferentes al de otro tipo de clases. Sin embargo, y a pesar de que el estilo expositivo - también llamado tradicional o de verificación — ha sido criticado por numerosos especialistas en educación científica (Hodson, 1994), todavía suele ser el predominante en las clases de laboratorio (Sánchez, Odetti y Lorenzo, 2016), por lo que también queda mucho por hacer en este campo.

A todas estas circunstancias los docentes universitarios hacen frente como pueden porque, en última instancia, son ellos quienes garantizan la calidad de los profesionales que egresan de sus aulas.

En resumen, la universidad constituye un recorte particular del objeto educativo, donde los contenidos cobran una singular importancia. Es una institución que debe afrontar los cambios sociales y una perplejidad cada vez más apremiante, una población de estudiantes heterogéneos pertenecientes a una nueva cultura de aprendizaje, una crisis en sus diseños curriculares, y un cuerpo de docentes con escasa o nula preparación para hacerle frente a todos estos desafíos.

Finalmente, podemos reconocer entonces que el nivel universitario en sí mismo se ha convertido en un campo que requiere incrementar los conocimientos sobre su propia naturaleza, desarrollo y desempeño, así como la naturaleza de los sujetos que allí participan y de las diversas interacciones que establecen los individuos entre sí, con la propia institución, con los campos disciplinares del saber, con los escenarios laborales y profesionales, y con la sociedad y el ambiente al que pertenecen.

\section{La formación de los docentes universitarios}

Hasta no hace mucho, los profesores universitarios y el sistema universitario en general parecían asumir que la formación pedagógica no era necesaria para desempeñarse como docentes (Jackson, 
2002). La situación más habitual era, y es, que los interesados en desempeñarse como docentes se incorporaran como asistentes o ayudantes (teacher assistants) en una determinada cátedra o departamento docente, y dieran sus primeros pasos colaborando con algún docente más experimentado. En un principio asumiendo tareas de baja responsabilidad, comúnmente asociadas a las clases de trabajos prácticos, para pasar luego a cumplir tareas de mayor exigencia como hacerse cargo de la explicación de algún pequeño tema, hasta que finalmente la autoridad de la cátedra lo considerara con la suficiente práctica y pericia para realizar algún tipo de evaluación de sus alumnos. De esta manera, lo más habitual es que la carrera como docente universitario se vaya construyendo a partir de la propia experiencia individual, al principio como estudiante y luego como colaborador de otros docentes, a la que se le agregarían sus propias capacidades personales para la imitación o confrontación con los otros docentes de su grupo, la autogestión para el aprendizaje y sus inquietudes para la actualización y el perfeccionamiento. Este proceso suele demorar años, y se desarrolla de una manera intuitiva y solitaria, lejos de una estructura formal que le ofrezca al docente en formación un andamiaje en donde anclar las experiencias que recoge de sus clases, sin un marco teórico que le permita conceptualizar las diversas situaciones en las que se ve inmerso, ni posibilidades para analizar ni reflexionar sobre su propia práctica.

En aquellos casos en los que aparece algún tipo de propuesta más o menos formalizada (por ejemplo, cursos de ayudantes) (Lorenzo et al., 2012), esta se orienta a los contenidos netamente disciplinares, plasmados como una profundización de los tópicos que se deben enseñar a los alumnos de la carrera o una extensión de los mismos, incorporando más ejemplos o nuevos temas propios de la disciplina. Sin embargo, en muy escasas ocasiones, pueden encontrarse en estas propuestas dispositivos orientados a la enseñanza de esos tópicos al alumnado de esa carrera. Esto responde claramente a una concep- ción de la enseñanza basada en la lógica disciplinar, a pesar de que ya ha sido sobradamente cuestionada desde hace tiempo (Hernández y Sancho, 1993).

Por fortuna, en las últimas décadas han comenzado a aparecer diferentes dispositivos de capacitación que con mayor o menor éxito propenden al desarrollo de las competencias docentes en la universidad (Tejeda, 2009; Zabalza, 2007).

En una sociedad como la nuestra, como hemos expuesto, debemos estar preparados para enfrentarnos de manera permanente a nuevas situaciones, cambios e incertidumbre, que implican necesariamente un aprendizaje a lo largo de toda nuestra vida (life long learning). Esta sociedad, que cada vez nos exige más a niveles intelectuales, personales y sociales, hace que debamos ser capaces de seguir aprendiendo por nosotros mismos de manera autónoma.

Estas nuevas metas de aprendizaje se aplican tanto para los estudiantes como para los docentes porque, en definitiva, se trata de ser competentes. Según Pozo y Monereo (2007), ser competente es poseer un conocimiento funcional, no inerte, utilizable y reutilizable, que pueda ser aplicado en diferentes problemas reales, en nuevas o viejas tareas, en nuevos contextos que supongan ir más allá de lo aprendido. Básicamente, podemos referirnos a cuatro escenarios de actuación en donde deberemos desplegar nuestras competencias. Estos son los escenarios educativo, profesional y laboral; social, y afectivo y emocional. En cada caso, deberemos recurrir a un conjunto de recursos potenciales para saber qué, cómo, cuándo, para qué y por qué, a fin de enfrentarnos a una situación concreta.

Cada carrera universitaria y cada desempeño profesional poseen un conjunto de competencias que le son propias y distintivas. Sin embargo, existen también un conjunto de competencias básicas que son comunes. Por ejemplo, las competencias personales vinculadas a la autonomía y a la iniciativa personal; las competencias sociales que se re- 
lacionan con el conocimiento y la interacción con el mundo físico y social, así como con el desempeño de la ciudadanía; y, las competencias relativas al uso de herramientas de todo tipo que incluyen competencias comunicativa-lingüística, matemática, digital, artística, cultural, y la competencia de aprender a aprender.

Muchas veces, estas competencias han sido descritas pensando únicamente en los alumnos, y está muy bien. Pero tal vez debido a que los profesores universitarios no suelen considerarse a sí mismos como alumnos, no se hayan detenido a pensar en que ellos también requieren desarrollar competencias, no solo como profesionales (ingenieros, médicos, químicos) o investigadores científicos (que es todo un tema en sí mismo), sino y fundamentalmente, sus competencias como profesionales docentes.

Es por estas razones que la formación pedagógica de los docentes universitarios no debe pensarse a la ligera, dado que por ser un colectivo de características muy particulares, requiere de un diseño notablemente diferente al de otros dispositivos didácticos.

- En primer lugar, los docentes universitarios están sometidos a la cultura universitaria de la cual forman parte. En muchas ocasiones, sus obligaciones trascienden las de enseñanza y su labor se completa con actividades de investigación y de extensión. Estas últimas, pero fundamentalmente las vinculadas a la investigación y sus requisitos, suelen ser muy rígidas y son las que a menudo se valoran de manera preferencial para la promoción en la carrera académica por encima de los desempeños en la enseñanza.

- $\quad$ En segundo lugar, y a diferencia del joven estudiante en busca de un título profesional de grado, los docentes universitarios, al convertirse en "alumnos de la formación", ya son adultos en pleno ejercicio de su adultez. Son graduados universitarios, muchos de ellos con una formación de posgrado, maestría o doctorado, al- gunos con muchos años de experiencia como docentes en la universidad y, tal vez, hasta se hayan desempeñado en cargos de decisión y autoridad. Otros, acaso, ocupen cargos de elevada responsabilidad en sus campos profesionales, o sean reconocidos dentro del sistema científico.

- Como un tercer rasgo para tener en cuenta, estos alumnos suelen tener muchos años de experiencia como docentes en el terreno y, por tanto, han desarrollado ciertos hábitos, conductas y teorías, mayormente implícitas, que les han permitido subsistir en la actividad.

- Por último, un aspecto que no debe despreciarse, es que así como los docentes universitarios son expertos en un área determinada de conocimiento, no lo son en cuestiones relacionadas con las teorías, los modelos y el vocabulario de la didáctica y la pedagogía, situación que los sensibiliza y los obliga a enfrentar sus propias limitaciones.

Hasta nuestros días, no conocemos que exista una formación pedagógica formal y obligatoria previa al ejercicio de la docencia universitaria, como existe para otros niveles del sistema educativo. En consecuencia, debemos pensar, configurar y ofrecer propuestas exclusivas para este colectivo, considerándola como una formación de cuarto ciclo o de cuarto orden. Desde nuestra perspectiva (Lorenzo, 2012), debido al peculiar recorte de contenidos que requiere una carrera universitaria, la formación del profesorado universitario debería estar orientada al ejercicio de la docencia en los campos disciplinares específicos. Sin embargo, las propuestas dirigidas a este grupo de profesionales de la docencia suelen estar organizadas en dos dimensiones paralelas. Por un lado, se les ofrecen instancias de actualización disciplinar, a cargo de especialistas en el campo; $y$, por otro, algunos cursillos o talleres pedagógicos a cargo de especialistas en educación, pero alejados de la disciplina de los formandos. Esto conlleva la 
falsa creencia o expectativa de que, una vez completados ambos trayectos, el sujeto será capaz de integrar de manera efectiva lo aprendido en ambos (tal vez recurriendo a ciertos conjuros que no pudieron lograrse ni siquiera en la elaboración de la propuesta formativa). Está claro que propuestas de ese tipo no son suficientes para la formación de los docentes dado que su desarrollo profesional (Imbernón, 1994), por tratarse de una actividad compleja, variada y variable, no puede pensarse alejado de la integración entre instancias de reflexión que atiendan tanto a los aspectos personales, disciplinares, contextuales como a la propia práctica (Vázquez, Jiménez y Mellado, 2007).

Pensando especialmente en la formación pedagógica de los docentes universitarios (Valcárcel, 2005) y de nivel superior, ofrecemos una propuesta de enseñanza que bien podría denominarse blending teaching, es decir, una propuesta que combine de manera integrada el conocimiento disciplinar (química, física, biología) y los conocimientos pedagógicos para poder modelizar de manera efectiva la enseñanza de la química, de la física o de la biología, ilustrando con ejemplos disciplinares distintas opciones de clasificación, selección y secuenciación de contenidos; planificación y diseño de ejercicios, problemas, trabajos prácticos de laboratorio; con la implementación de enfoques que incluyan los aportes de los modelos de ciencia-tecnología-sociedad (CTS) y los de las disciplinas metacientíficas (Historia y Epistemología de la Ciencia), por mencionar algunos. A nuestro entender, este docente ha de haber transitado un recorrido personal en ambos campos de conocimiento. De este modo, conocería los obstáculos y las dificultades en cada caso particular y, también, aquellos que devienen de la combinación de ambos campos, de manera tal que pueda enseñar enseñando (Lorenzo, 2012), seleccionando los ejemplos, las analogías y las actividades con el fin de desarrollar los modelos didácticos más apropiados para cada caso.
Para completar la idea, sería conveniente basar la propuesta de aprendizaje en el modelo de blending learning, que combina actividades presenciales con un trabajo sostenido a través del aula virtual u otros dispositivos similares. Además, el eje vertebrador debe responder a las necesidades reales de los cursantes (que no necesariamente coinciden con sus demandas), a fin de ofrecer una propuesta flexible, motivante y útil que se adapte a los escenarios concretos de actuación y respete las múltiples responsabilidades de estos particulares alumnos. Durante los encuentros presenciales se debería lograr implementar un trabajo experiencial, donde los cursantes pudieran desempeñar alternativamente dos roles complementarios, el de todo aquel que se inscribe en un curso de formación o capacitación, el rol de alumno; y otro rol, como docente-evaluador, que le permita reflexionar de manera crítica sobre su propio aprendizaje, y sobre la propuesta de enseñanza del curso.

Así, en ciertos momentos, la recuperación del rol de alumno es fundamental para que puedan redescubrir la emoción ante lo desconocido, tomar conciencia sobre las propias limitaciones, enfrentar las dificultades ante tareas novedosas, recuperar las estrategias de lectura para el estudio y, sobre todo, esa perspectiva estudiantil que afronta por primera vez un mundo poco conocido, desafiante y, en algunos casos, no del todo estimulante. Por otra parte, y dada su experiencia como docentes universitarios, que implica, como ya hemos dicho, tareas de investigación y de evaluación además de la enseñanza, ese otro rol permite recuperar esa mirada crítica y reflexiva que promueva la búsqueda de sentido a las prácticas pedagógicas, ajenas y propias, que permita comprenderlas a la luz de los marcos teóricos disponibles, que fomente el análisis de las diferentes situaciones, y la evaluación y el desarrollo del metaconocimiento. En este doble juego de la formación y la investigación sobre la propia práctica, resulta decisivo la incorporación de los aportes provenientes de las disciplinas metacientíficas a los dispositivos formativos (Lorenzo y Farré, 2014). 
Esta propuesta de concebir la formación del profesorado universitario se enmarca en el modelo del conocimiento didáctico del contenido (CDC, más conocido como PCK, por sus siglas en inglés, Pedagogical Content Knowledge) (Farré y Lorenzo, 2009), propuesto por Lee Shulman (1986). Surgido inicialmente como modelo de investigación que proponía recuperar el paradigma perdido del conocimiento disciplinar en la enseñanza, ha fructificado como un modelo potente para ser aplicado en las aulas. El modelo del PCK es actualmente tanto un marco teórico, desde donde desarrollar la investigación para documentar el conocimiento profesional de los profesores, como una estrategia versátil para contribuir al desarrollo de ese mismo conocimiento profesional. Este modelo ha tenido un alto impacto en todo el mundo (Abell, 2008; Barnett y Hodson; 2001; Carlson y Gess-Newsome, 2013; Gess-Newsome y Lederman, 1999; Berry, Loughran y van Driel, 2008; Kind, 2009; Loughran, Mulhall y Berry, 2012; Park, Jang, Chen y Jung, 2011; Park y Oliver, 2008). En el contexto latinoamericano, fue nuestro querido y reconocido profesor Andoni Garritz, quien impulsó este campo de investigación desde muy diversas aristas, tanto para la formación de profesores (Garritz, 2006) como en las líneas de vanguardia vinculadas a la afectividad (Garritz, 2010), siendo uno de sus últimos trabajos la compilación de escritos sobre el PCK desarrollados en la región, en nuestros contextos (Garritz, Daza y Lorenzo, 2014).

El conocimiento didáctico del contenido fue descrito como una amalgama entre el conocimiento disciplinar y el pedagógico, y constituye un tipo particular de conocimiento propio de cada profesor, para cada contenido, para un grupo determinado de estudiantes. Pero a pesar de su especificidad, ha demostrado una gran funcionalidad como estrategia pedagógica versátil para la formación docente (González y Rossi, 2014), porque permite la gestión un conocimiento transferible al interior del aula (Bucat, 2004).

\section{Algunos modelos para el estudio de las prácticas docentes en el nivel superior}

Lo que ocurre en el contexto del aula cuando docentes y alumnos interactúan con el propósito de construir conocimiento de forma compartida (Coll y Onrubia, 1996; Edwards y Mercer, 1994) es un objeto difícil de abarcar desde la investigación didáctica por ser un sistema dinámico, multidimensional y variable. Es durante la clase cuando profesores y estudiantes interactúan con los contenidos del currículo, la institución y las políticas educativas, la tecnología disponible, la sociedad y la cultura en la que están inmersas. En este contexto de interacción, la enseñanza es concebida como la actividad intencional que el docente desarrolla con el fin de que sus alumnos aprendan el contenido que está enseñando. Las prácticas educativas son, por tanto, un escenario rico y complejo que en los últimos tiempos han comenzado a ser estudiadas por los enfoques socioculturales y situados de la cognición, el aprendizaje y la enseñanza (Coll y Sánchez, 2008).

Desde esta perspectiva, el intercambio de información en clase debería dar lugar a una construcción activa de conocimiento formal por parte del alumno, y favorecer la apropiación de los saberes disciplinares y, por tanto, está claro que la resignificación de los contenidos enseñados en clase resulta imprescindible para el aprendizaje. Generalmente, en los niveles primario y secundario la enseñanza se lleva a cabo a partir de la negociación y la consecuente construcción de significados compartidos entre el docente y sus alumnos. Por tanto, la coconstrucción de conocimientos se evidencia en el diálogo y en las intervenciones de los participantes en el aula. Sin embargo, en el caso del nivel superior, el diálogo es escaso y predomina el monólogo del profesor. Ante tal situación, la negociación y resignificación de los contenidos se vería obstaculizada por la propia dinámica de la clase, en la cual el rol del docente es preponderante y es quien guía e imparte las explicaciones. Asimismo, las interacciones que ocurren en clases universitarias de 
ciencias encierran al menos dos aspectos que las distinguen de otros niveles educativos, estos son el elevado nivel de abstracción de los contenidos que abordan y el uso de diferentes sistemas simbólicos no solo para representar conceptos, sino y especialmente para la construcción de nuevos conocimientos.

Debido a los nuevos perfiles estudiantiles, y los cambios sociales y estructurales que hemos comentado, el nivel superior ha comenzado a llamar la atención como objeto susceptible de ser abordado por las investigaciones en didáctica, tanto por aquellas de carácter general como por las didácticas específicas que consideran muy especialmente las interacciones entre el docente y los estudiantes con los contenidos disciplinares (Adúriz-Bravo e Izquierdo-Aymerich, 2002). En este sentido, se ha generado una necesidad a la cual la investigación en didáctica de las ciencias intenta dar respuestas llevando adelante proyectos de investigación que analicen, estudien y reflexionen sobre cada uno de los elementos y de las múltiples interacciones que surgen en las prácticas educativas en la universidad, entendidas estas en sentido amplio, de modo de proporcionar nuevos conocimientos y estrategias que puedan ser transferidos e implementados en las aulas.

En nuestro grupo de trabajo, hace ya varios años que venimos estudiando las prácticas educativas en el nivel universitario en carreras de ciencias, principalmente para las asignaturas de Química Orgánica y Física. Los modelos teóricos que hemos adoptado para abordar esta tarea se enmarcan en dos grandes líneas: aquellos que se refieren al análisis del discurso de los profesores y el modelo del conocimiento didáctico del contenido.

Ya hemos definido al conocimiento didáctico del contenido y su aplicabilidad en la formación docente. Ahora nos detendremos a considerar su potencialidad como modelo de investigación dado que se trata de un campo de trabajo en plena expansión porque recupera la importancia del contenido disciplinar a la hora de pensar sobre las prácticas educativas. El estudio y la documentación del PCK conllevan algunas dificultades metodológicas, precisamente porque se modifican durante el proceso de recolección en el campo; situación que, por otro lado, es la que habilita su uso como estrategia formadora. Desde que fue propuesto por Shulman (1986), las investigaciones sobre el PCK se han basado primordialmente en cuestionarios escritos y entrevistas (Baxter y Lederman, 1999) sobre poblaciones de profesores en formación. A pesar del tiempo transcurrido son muy pocas las referencias en la literatura a la documentación del PCK en profesores de ciencias naturales y experimentales en el nivel superior.

Nuestras propias investigaciones han puesto de manifiesto que la metodología que podríamos definir como tradicional para la documentación del PCK (Loughran, Mulhall y Berry, 2008) es especialmente útil para la detección del conocimiento declarativo de los profesores donde se manifiestan las ideas explícitas (Farré y Lorenzo, 2008). Sin embargo, no permite indagar ideas más implícitas, menos mediadas por el discurso compartido de la comunidad educativa o, como en el caso de los docentes universitarios, permite sobrellevar su falta de dominio del vocabulario pedagógico (Jackson, 2002). Por ello, ante la necesidad de implementar nuevas estrategias para investigar las prácticas educativas en el nivel universitario con una mirada holística y sistémica, desarrollamos una metodología que combina el tradicional cuestionario de representación de contenido con el análisis del discurso (Lorenzo y Farré, 2009; Farré y Lorenzo, 2014).

Esta estrategia metodológica se basa en el rol central que desempeña el uso de la palabra como recurso de los docentes. Especialmente en el nivel universitario el discurso del profesor sigue ocupando un lugar preponderante, independientemente del tipo de clase; ya sea esta de tipo teórico, de resolución de problemas o de laboratorio; o de la posibilidad de utilizar otros recursos, como los que brindan las nuevas tecnologías. Durante las clases, el verbo es acompa- 
ñado por otros recursos semióticos como las representaciones externas y el lenguaje corporal y gestual (Lemke, 2002) que configuran un discurso particular.

En el caso de los profesores de ciencias, el discurso docente resulta una mezcla de lenguaje coloquial y científico para referirse tanto a lo observable a través de los sentidos, como a los pensamientos, procedimientos y teorías de la ciencia. Estos lenguajes dependen de la disciplina, poseen sus propias reglas y se caracterizan por la rigurosidad con que definen sus términos técnicos, a la vez que sirven como sistema de recursos para la creación de nuevos significados (Schummer, 1998). Dicho de modo general, el lenguaje científico es, por tanto, un instrumento para pensar, crear y comunicar conceptos, métodos y metas que trasciende al lenguaje cotidiano. Por consiguiente, cuando un profesor desarrolla determinados contenidos en clase emplea un lenguaje condicionado por el modelo teórico que explícita o implícitamente adopta. Para los profesores, la representación de conceptos con fórmulas u otros sistemas notacionales resulta esclarecedora; sin embargo, para la mayoría de los estudiantes que recién se inician en el estudio de estas disciplinas, generalmente constituye un obstáculo (Lorenzo y Rossi, 2009). En síntesis, el discurso en clase del profesor es extremadamente rico en información específica, y está altamente contextualizado dado que se asocia a una determinada situación de clase. De tal manera que a partir del análisis del discurso (Candela, 2001), y dada la relación entre lo que los profesores piensan y cómo enseñan (Coll y Sánchez, 2008), puede evidenciarse la forma de pensar del profesor sobre el objeto de conocimiento, sobre el aprendizaje y la enseñanza. Es decir, los componentes implícitos de la práctica docente (Kane, Sandretto y Heath, 2002) conocidos como creencias o concepciones docentes. Estos elementos pueden ser evidenciados a partir del estudio del discurso del profesor en clase que informa sobre el conocimiento didáctico del contenido en uso sobre el tópico que está enseñando.
Pero, como hemos dicho, teniendo en cuenta la complejidad del sistema en estudio, seguramente estos dos modelos no agotan las posibilidades de investigación en el campo y todavía quedan muchas preguntas que buscan respuestas. Para ejemplificar ofrecemos algunos interrogantes en esta línea que, creemos, podrían orientar la investigación en este campo:

¿Qué tipo de formación requiere un docente universitario? ¿Cuáles contenidos son los necesarios y suficientes para garantizar un sólida formación profesional de base? ¿Qué tipo de orientación curricular se adapta mejor a cada carrera universitaria? ¿Qué relación guarda o debería guardar la formación universitaria con el contexto social de pertenencia? ¿A qué se debe el elevado nivel de abandono de estudiantes de las carreras científicas? ¿Cómo favorecer el tránsito curricular positivamente promoviendo aprendizajes genuinos?

¿Cómo hace el profesor del nivel superior para asegurarse de que está comunicando lo que quiere enseñar? ¿Cuáles son las estrategias más efectivas para la enseñanza de su asignatura? ¿Cómo enfrentan o podrían enfrentar los docentes universitarios las dificultades de aprendizaje de sus estudiantes? ¿Cuál es la relación entre el desempeño académico y el desarrollo de competencias profesionales en los estudiantes?

\section{Conclusiones y perspectivas}

A lo largo de este texto hemos planteado la necesidad de incorporar las prácticas educativas de nivel superior como objeto de estudio de la didáctica de las ciencias. Hemos ofrecido algunas reflexiones sobre los motivos que nos llevan a enunciar que la enseñanza y el aprendizaje en la universidad son, hoy en día, un tema que debería estar incluido en las agendas de investigación educativa de diversas áreas de conocimiento, particularmente en el campo de la didáctica de las ciencias naturales. También hemos ofrecido algunos ejemplos de modelos teóricos que 
pueden resultar de utilidad para la investigación en este campo. Al mismo tiempo, hemos intentado poner en debate la formación pedagógica de los profesores que se desempeñan en el nivel superior, considerando que su rol como enseñantes no está exento de una adecuada formación profesional.

Afortunadamente, cada vez son más los grupos que comienzan a desarrollar investigaciones sobre este nivel, y también han ido creciendo las universidades que ofrecen trayectos formativos específicos para los docentes universitarios, así como la posibilidad de desarrollar carreras de posgrado en la temática. En estos espacios es necesario tener presentes cuestiones que, aunque son comunes a otros niveles educativos, no pueden dejarse de lado:

- La reflexión recurrente sobre las diferentes actividades de enseñanza y de aprendizaje de las ciencias, con la incorporación de los aportes de las disciplinas metacientíficas.
- $\quad$ La revisión permanente de los avances, resultados y controversias de las líneas actuales de investigación en didáctica de las ciencias en relación con las prácticas de enseñanza.

- $\quad$ El ejercicio de las competencias para el metaanálisis de la clase como estrategia para la reflexión y la comprensión de la propia práctica.

- $\quad$ El establecimiento de redes y equipos de trabajo que faciliten la construcción colectiva de nuevo conocimiento a través de la formación de recursos humanos con el fin de formalizar becas, tesis de maestría y de doctorado.

Sin excepción, el camino que se recorre por las aulas universitarias ofrece una experiencia única de aprendizaje que transforma al sujeto que lo transita de una manera permanente. Contribuir en cada uno de los recorridos individuales a fin de promover las mejores experiencias de aprendizaje y de desarrollo humano y profesional es el gran desafío de la universidad de estos tiempos.

\section{Referencias}

Abell, S. K. (2008). Twenty years later: Does pedagogical content knowledge remain a useful idea? International Journal of Science Education, 30 (10), 1405-1416.

Adúriz-Bravo, A. e Izquierdo-Aymerich, M. (2002). Acerca de la didáctica de las ciencias como disciplina autónoma. Revista Electrónica de Enseñanza de las Ciencias, 1 (3), 130-140.

Barnett, J. y Hodson, D. (2001). Pedagogical context knowledge: Toward a fuller understanding of what good science teachers know. Science Education, 85 (4), 426-453.

Baxter, J. A. y Lederman, N. G. (1999). Assessment and measurement of PCK. En Gess-Newsome, J., Lederman, N. G. (eds.). Examining Pedagogical Content Knowledge (pp. 147-162). Dodrecht: Kluwer Academic Publishers.

Berry, A., Loughran, J. y van Driel, J. H. (2008). Revisiting the roots of pedagogical content knowledge. International Journal of Science Education, 30 (10), 1271-1279.

Bucat, R. (2004). Pedagogical content knowledge as a way forward: Applied research in chemistry education. Chemistry Education: Research y Practice, 5 (2), 215-228. 
Candela, A. (2001). Corrientes teóricas sobre discurso en el aula. Revista Mexicana de Investigación Educativa, 6 (12), 317-333.

Carlson, J. y Gess-Newsome, J. (2013). The PCK summit consensus model and definition of pedagogical content knowledge. En Symposium “Reports from the Pedagogical Content Knowledge (PCK) Summit”, ESERA Conference 2013, September. Recuperado de http://www.esera2013.org.cy/programme/detailedprogramme/parallel-sessions/?session=15

Coll, C. y Onrubia, J. (1996). La construcción de significados compartidos en el aula: actividad conjunta y dispositivos semióticos en el control y seguimiento mutuo entre profesor y alumnos. En Coll, C. y Edwards, D. Enseñanza, aprendizaje y discurso en el aula. Aproximaciones al estudio educacional. Madrid: Alianza Aprendizaje.

Coll, C.y Sánchez, E. (2008). Presentación. El análisis de la interacción alumno-profesor: líneas de investigación. Revista de Educación, 346, 15-32.

Edwards, D.y Mercer, N. (1994). El conocimiento compartido: el desarrollo de la comprensión en el aula. Barcelona: Paidós.

Farré, A. S. y Lorenzo, M. G. (2008). La formación docente de los profesores universitarios de ciencias. Aportes desde la investigación en didáctica de las ciencias. En Jiménez Liso, M. R. (ed.). Actas de los XXIII Encuentros de Didáctica de las Ciencias Experimentales (pp. 625-632). Universidad de Almería: Almería.

Farré, A. S. y Lorenzo, M. G. (2009). Conocimiento pedagógico del contenido: una definición desde la química. Educación en la Química, 15 (2), 103-113.

Farré, A. S. y Lorenzo, M. G. (2014). El escurridizo conocimiento didáctico del contenido: estrategias metodológicas para su documentación. En Lorenzo, M. G., Daza, S. y Garritz, A. (eds.) (2014). Conocimiento didáctico del contenido. Una perspectiva Iberoamericana. Saarbrücken: Académica Española.

Garritz, A. (2006). Historia y retos de la formación de profesores (Algo más sobre Lee Shulman). Educación Química, 17(3), 322-326.

Garritz, A. (2010). Pedagogical content knowledge and the affective domain of scholarship of teaching and learning. International Journal for the Scholarship of Teaching and Learning, 4 (2), 1-6. Recuperado de http:// digitalcommons.georgiasouthern.edu/cgi/viewcontent.cgi?article=1246andcontext=ij-sotl

Garritz, A., Daza, S.y Lorenzo, M. G. (ed.) (2014). Conocimiento didáctico del contenido. Una perspectiva iberoamericana. Saarbrücken: Académica Española.

Gess-Newsome, J.y Lederman, N. G. (eds.) (1999). Examining Pedagogical Content Knowledge. The Construct and its Implications for Science Education. Dordrecht: Kluwer.

González, N. y Rossi, A. (2014). La enseñanza de la meiosis en el nivel secundario: el conocimiento didáctico del contenido de profesores expertos y principiantes. En Garritz, A., Daza, S. y Lorenzo, M. G. (ed.) (2014). Conocimiento didáctico del contenido. Una perspectiva iberoamericana (pp. 403-453). Saarbrücken: Editorial Académica Española. 
ISSN $0123-1294$ | e-ISSN 2027-5358 | Educ.Educ. Vol. 20. No. 2 | Mayo-agosto de 2017 | pp. 249-263.

Universidad de La Sabana | Facultad de Educación

Hernández, F.y Sancho, J. (1993). Para enseñar no basta con saber la asignatura. Barcelona: Gedisa.

Hodson, D. (1994). Hacia un enfoque más crítico del trabajo de laboratorio. Enseñanza de las ciencias, 12 (3), 299-313.

Hofstein, A. y Mamlok-Naaman, R. (2007). The laboratory in science education: The state of the art. Chemistry Education: Research and Practice, 8 (2), 105-107.

Imbernón, F. (1994). La formación y el desarrollo profesional del profesorado. Hacia una nueva cultura profesional. Barcelona: Graò.

Jackson, P. W. (2002). Práctica de la enseñanza. Avellaneda: Amorrurtu.

Kane, R., Sandretto, S.y Heath, C. (2002). Telling half the story: A critical review of research on the teaching beliefs and practices of university academics. Review of Educational Research, 72 (2), 177-228.

Kind, V. (2009). Pedagogical content knowledge in science education: Perspectives and potential for progress. Studies in Science Education, 45 (2), 169-204.

Lemke, J. (2002). Enseñar todos los lenguajes de la ciencia: palabras, símbolos, imágenes y acciones. En Benlloch, M. (comp.). La educación en ciencias: ideas para mejorar su práctica (pp.159-186). Barcelona: Paidós.

Lorenzo, M. G. (2012). Los formadores de profesores: el desafío de enseñar enseñando. Profesorado: revista de currículum y formación del profesorado, 16 (2), 295-312.

Lorenzo, M. G. y Farré, A. S. (2014). Epistemología, historia y filosofía de las ciencias: un puente entre la investigación didáctica y la enseñanza de las ciencias (pp. 167-182). En Arellano, M., Merino, C. y Adúriz-Bravo, A. (eds.). Avances en didáctica de la Química: modelos y lenguajes. Chile: Pontificia Universidad Católica de Valparaíso.

Lorenzo, M. G. y Rossi, A. M. (2009). Análisis de las estrategias didácticas presentes en el discurso del profesor universitario de Química orgánica. En Campos Hernández, M. A. (comp.). Discurso, construcción de conocimiento y enseñanza (pp. 149-178). México: ISSUE, UNAM/Plaza y Valdés.

Lorenzo, M. G., Blanco, M., Caterina, C., Reverdito, A., Shmidt, S. y Salerno, A. (2012). Los cambios curriculares y su relación con la formación de nuevos docentes en el ámbito universitario. Educación en la Química, 18 (1), 99-108.

Lorenzo, M. y Farré, A. (2009). El análisis del discurso como metodología para reconstruir el conocimiento didáctico del contenido. Enseñanza de las Ciencias, Número Extra, 342-345. Recuperado de http://ensciencias.uab.es/congresoog/numeroextra/art-342-345.pdf

Loughran, J., Mulhall, P. y Berry, A. (2008). Exploring pedagogical content knowledge in science teacher education. International Journal of Science Education, 30 (10), 1301-1320.

Loughran, J. J., Mulhall, P.y Berry, A., (2012). Understanding and Developing Science Teachers'Pedagogical Content Knowledge (2 ed.). Rotterdam: Sense Publishers. 
Monereo, C. y Pozo, J. I. (2003). La universidad ante la nueva cultura educativa. Enseñar y aprender para la autonomía. Madrid: Síntesis.

Park, S.y Oliver, S. (2008). Revisiting the conceptualisation of pedagogical content knowledge: PCK as a conceptual tool to understand teachers as professionals. Research in Science Education, 38 (3), 261-284.

Park, S., Jang, J.-Y., Chen, Y.-C. y Jung, J. (2011). Is pedagogical content knowledge (PCK) necessary for reformed science teaching? Evidence from an empirical study. Research in Science Education, 41 (2), 245-260.

Pozo, J. I. y Monereo, C. (2007). Competencias para (con)vivir con el siglo XXI. Cuadernos de Pedagogía (monográfico), 370, 9-84.

Reid, N.y Shah, I. (2007). The role of laboratory work in university chemistry. Chemistry Education Research and Practice, 8 (2), 172-185.

Sánchez, G., Odetti, H. y Lorenzo, G. (2016). Caracterización de la práctica educativa de docentes universitarios en clases de laboratorio. En Membiela Iglesia, F. P. (ed.). La práctica docente en la enseñanza de las ciencias. Vigo: Educación Editora (en prensa).

Schummer, J. (1998). The chemical core of chemistry I: A conceptual approach. HYLE, An International Journal for the Philosophy of Chemistry, 4 (2), 129-162.

Seré, M. (2002). La enseñanza en el laboratorio. ¿Qué podemos aprender en términos de conocimiento práctico y de actitudes hacia la ciencia? Enseñanza de las Ciencias, 20(3), 357-368.

Shulman, L. S. (1986). Those who understand: knowledge growth in teaching. Educational Researcher, 15, 4-14.

Tejeda Fernández, J. (2009). Competencias docentes. Profesorado. Revista de currículum y formación del profesorado, 13 (2), 1-15. Recuperado de http://www.ugr.es/ recfpro/rev132COL2.pdf

Valcárcel, M. (2005) (coord.). La preparación del profesorado universitario para la convergencia europea en educación superior. Informe de investigación, Proyecto EA2003-0040.

Vázquez, B., Jiménez, R. y Mellado, V. (2007). El desarrollo profesional del profesorado como integración de la reflexión y la práctica. La hipótesis de la complejidad. Revista Eureka, 4 (3), 372-393.

Zabalza, M. A. (2007). Competencias docentes del profesorado universitario. Madrid: Narcea. 
